\title{
Biophoton Imaging Evaluation of the Process of Rheumatoid Arthritis in Rats
}

\author{
Chengming Xia ${ }^{1,2}$, Jinzhong $\mathrm{Li}^{1,2}$, Yi Yue ${ }^{1,2}$, Linhua Chen ${ }^{1,2}$, Jiapei Dai ${ }^{1,2}$ \\ ${ }^{1}$ Wuhan Institute for Neuroscience and Neuroengineering (WINN), South-Central University for Nationalities, \\ Wuhan, China; ${ }^{2}$ Department of Neurobiology, College of Life Sciences, South-Central University for Nationalities, \\ Wuhan, China
}

Correspondence to: Jiapei Dai, jdai@mail.scuec.edu.cn

Keywords: Rheumatoid Arthritis, Freund's Adjuvant, Biophoton, Biophoton Imaging, Rat

Received: August 24, $2021 \quad$ Accepted: October 5, $2021 \quad$ Published: October 8, 2021

Copyright $\odot 2021$ by author(s) and Scientific Research Publishing Inc.

This work is licensed under the Creative Commons Attribution-NonCommercial International License (CC BY-NC 4.0). http://creativecommons.org/licenses/by-nc/4.0/

\section{(c) (1) () O) Open Access}

\section{ABSTRACT}

Rheumatoid arthritis (RA) is a common form of chronic inflammatory arthritis, and it mainly causes the destruction of small joints. The development of this disease is a relatively secret and repeated process, and therefore early diagnosis and evaluation of the disease is usually difficult. In this study, an arthritis model was successfully induced by injecting complete Freund's adjuvant (CFA) into the toes of lower limbs of Wistar rats. Seven days after injection of CFA, obvious redness and swelling appeared at the toe joints of lower limbs accompanied by more sensitivity to thermal stimulation. Using the ultraweak biophoton imaging system (UBIS) established by us, the toe joint area of the lower limbs of rats was imaged 7 days after injection of CFA. It was found that the volar part of lower limbs of arthritis rats showed significantly higher biophoton emissions compared with the control group. The results of this study may provide a basis for further research and development of early diagnosis and assessment of lesion progression of rheumatoid arthritis.

\section{INTRODUCTION}

Rheumatoid arthritis (RA) is the most common chronic inflammatory disease [1], and its main clinical manifestations are symmetry persistent joint swelling and pain. It is also considered a syndrome, including extraarticular manifestations, such as rheumatoid nodules, pulmonary involvement or vasculitis, and systemic complications. With the progress of disease course, patients with RA will have limited joint function due to joint pain and stiffness [2-6], however, early diagnosis and evaluation of the disease process are usually difficult, and comprehensive evaluation needs to be combined with multiple detection methods. Therefore, it is of great clinical value to establish a simple technique for early diagnosis and evaluation of disease progress. 
Ultraweak photon emission (UPE), also known as biophoton, exists in almost all organisms with a spectral range of $200-800 \mathrm{~nm}$, and an intensity of $10^{2}-10^{3}$ photons $/\left(\mathrm{cm}^{2} \cdot \mathrm{s}\right)$ [7-10]. Biophoton emissions are directly related to the free radical activity in the process of oxidative metabolism, as well as the physiological and pathological changes of the body, whereas the process of inflammation is often accompanied by the increase of free radical activity. Therefore, the detection of biophoton emission has a certain value in the pathological diagnosis in animals and plants [11-16]. In this study, by detecting and analyzing the intensity of biophoton emissions in the toe joint area of lower limbs in rats, we strived to establish a biophoton imaging technology that can be applied to the early diagnosis and disease process evaluation in patients with arthritis.

\section{MATERIAL AND METHODS}

\subsection{Rat Model of RA}

Male Wistar rat (3 - 4 weeks, $62 \pm 5$ g) were purchased from Hubei Provincial Laboratory Animal Public Service Center (Wuhan, China) and housed under standard conditions (12-h light/dark cycle, room temperature $18^{\circ} \mathrm{C}-25^{\circ} \mathrm{C}, 40 \%-50 \%$ humidity) with access to food and water ad libitum. The protocols were approved by the committee on the Ethics of Animal Experiments of South-Central university for Nationalities. Ten rats were divided into two groups ( 5 in each group and numbered). Complete Freund's adjuvant (CFA) was injected subcutaneously in the plantar part of the right hind paw of one group and the left hind paw of the other group, and the contralateral hind paw was set as the control. The dose of the first injection was $0.25 \mathrm{ml} / 100 \mathrm{~g}$ and the control was injected with the same dose of normal saline. The changes in diet and activity and the state of lower limb joints of rats in two groups were observed every day.

\subsection{Pain Sensitivity Test}

Heat sensitivity of the rat hind paw was determined using a Plantar Test Apparatus (ZH-200, Zhenghuabiologic, China). Rat was placed in a plastic box with a glass floor. After a 3-min habituation period, the plantar surface of hind paw was exposed to a beam of radiant heat through the glass floor. The baseline latencies were averaged over 4 trials, separated by a 10-min interval. The test was carried out once a day. A total of 17 tests were conducted, including the first one before CFA injection.

\subsection{Biophoton Imaging}

The rat's hind paw was imaged with UBIS according to the previous report [17]. The rat was fixed on a device and then put into the dark box of UBIS. The biophoton imaging was carried out with an EMCCD. The specific imaging parameters were as follows: 1 ) EM-CCD cooling temperature is $-95^{\circ} \mathrm{C} ; 2$ ) $1200 \times$ gain; 3 ) the exposure time is $900 \mathrm{~s}$ for each frame image; 4 ) the time course of imaging is $1.5 \mathrm{~h}$.

\subsection{Image Processing and Data Analysis}

A series of images were processed using the previously reported methods [17], including the removal of bright spots caused by the cosmic radiation, and the extraction of gray values from the region of interesting (ROI), such as the plantar joint of the hind limb of rat and background area.

$$
\begin{gathered}
\text { Relative gray values }(\mathrm{RGVs})=\text { the gray values of the region of interesting (ROI) } \\
- \text { the gray values of background area. }
\end{gathered}
$$

\subsection{Statistics}

Statistical analyses were performed using Microsoft Excel and two-tailed paired T-test was used to compare the differences between the treated group and control group. Probability values of $p<0.05$ were regarded as significant. 


\section{RESULTS}

\subsection{CFA-Induced Rat Model of RA}

During the experiment, except that the experimental group had different degrees of claudication in the lower limbs after injection of CFA for a period of time, no obvious behavioral abnormalities and weight changes were found in two groups. Seven days after injection of CFA, the plantar joint of rat hind limb presented swelling (Figure 1(A)), whereas the hind paws in controls had no such phenomenon (Figure 1(B)).

\subsection{Changes in Nociceptive Threshold}

As shown in Figure 2, there was no difference between the plantar part of the right and left hind paw in two groups before CFA injection. Heat hyperalgesia was found in the CFA injection hind paw lasting from the second day after CFA injection to the 16th days. The most obvious pain sensitive reaction was at the 7 th day after injection. A slow recovery process occurred until 12th day, and then there was a period of increased sensitivity.

The dynamic changes in the reaction time of the hind paw both in RA and control groups.
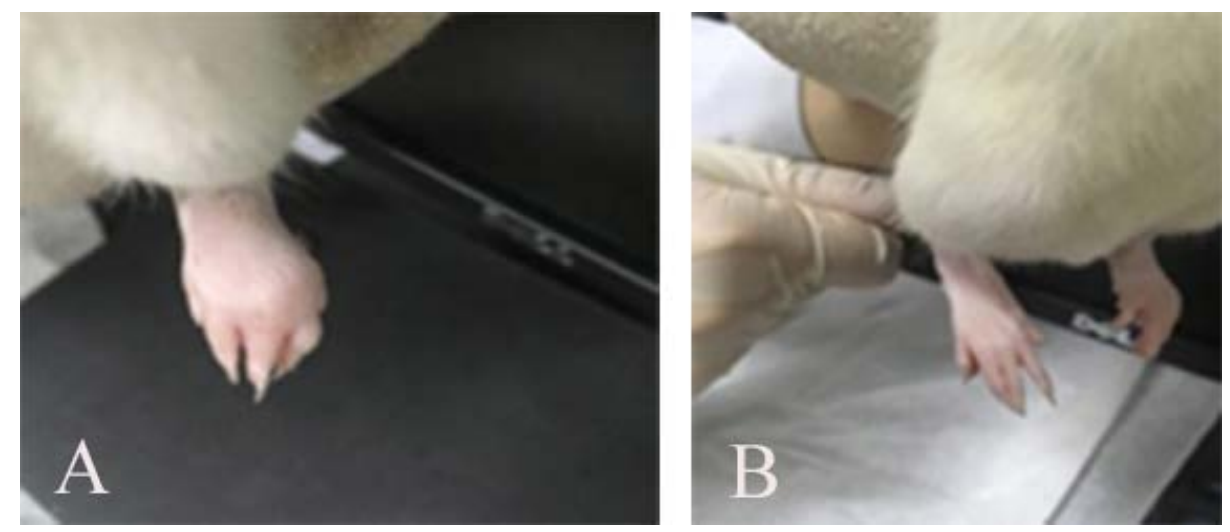

Figure 1. The changes in CFA-induced rat model of RA. (A) A representative regular image of the RA rat's hind paw, showing obvious swelling 7 days after injection of CFA; (B) A representative image of the normal rat's hind paw.

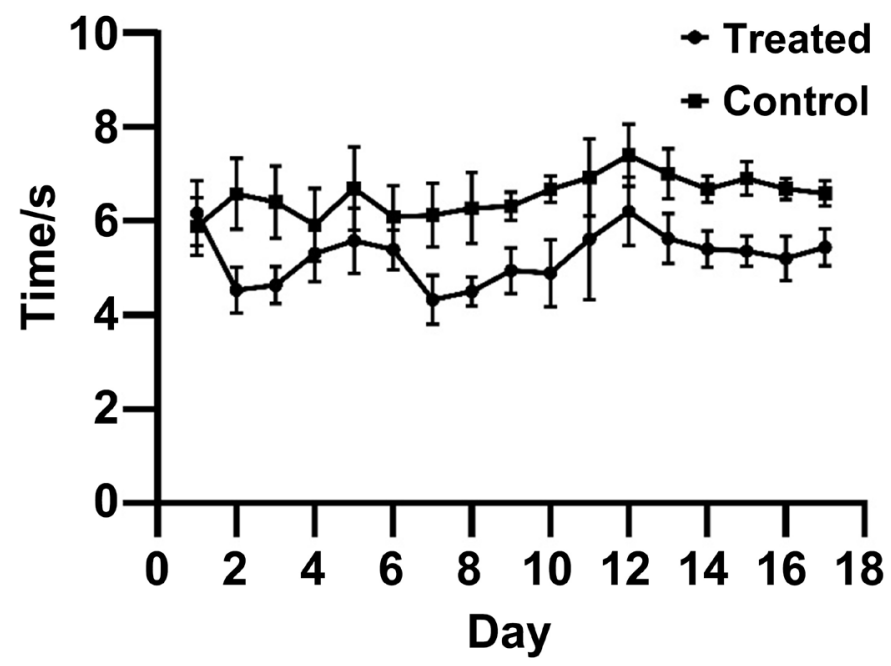

Figure 2. The hind paw reaction time induced by thermal stimulus. 


\subsection{Biophoton Emissions Were Higher in RA}

A swollen hind paw picture (Figure 3(A)) in a CFA treated rat corresponds to an image of biophoton imaging (Figure 3(B)). The regular picture and the image of biophoton imaging in a control rat hind paw are shown in Figure 3(C) and Figure 3(D), respectively. There was a significant decline of biophoton emission during the first $30 \mathrm{~min}$, then a slightly decay was observed within the next $60 \mathrm{~min}$. The biophoton emissions were significantly higher in the CFA treated paws than that in the controls at the different time points (Figure 3(E), Table 1, $\mathrm{n}=7$ ).

Table 1. Comparison of relative gray values between the CFA treated and control hind paws at the different time points.

\begin{tabular}{cccc}
\hline Time (min) & Treated & Control & $P$ value \\
\hline $\mathbf{1 5}$ & $104.8 \pm 37.90$ & $84.54 \pm 23.78$ & 0.29 \\
$\mathbf{3 0}$ & $55.71 \pm 14.39$ & $34.62 \pm 9.86$ & $0.012^{* *}$ \\
$\mathbf{4 5}$ & $42.33 \pm 7.52$ & $26.11 \pm 6.64$ & $0.0019^{* * *}$ \\
$\mathbf{6 0}$ & $36.56 \pm 3.87$ & $20.18 \pm 4.32$ & $0.000016^{* * *}$ \\
$\mathbf{7 5}$ & $29.85 \pm 4.94$ & $17.45 \pm 5.07$ & $0.0011^{* *}$ \\
90 & $29.67 \pm 5.32$ & $15.13 \pm 6.79$ & $0.0014^{* *}$ \\
\hline
\end{tabular}

Asterisks indicate a significant difference, $\mathrm{n}=7,{ }^{* *} p<0.01 ;{ }^{* *} p<0.001$.
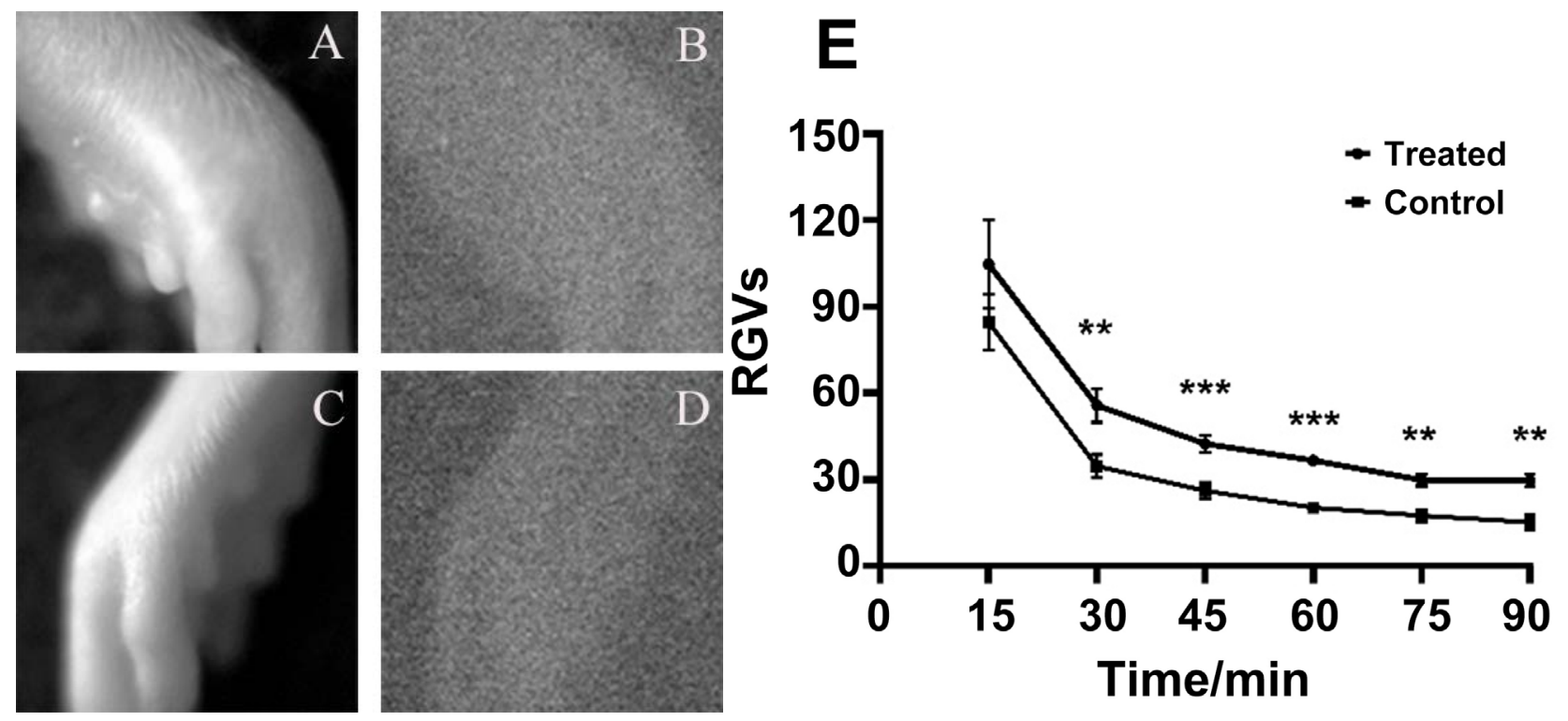

Figure 3. Biophoton imaging of hind paw in rats treated with CFA and saline. (A) A representative regular image of rat hind paw treated with CFA; (B) The corresponding image of biophoton imaging to A, showing obvious biophoton emissions in the region of hind paw (bright area). (C) A representative regular image of control rat hind paw. (D) The corresponding image of biophoton imaging to $\mathrm{C}$, showing relative weak biophoton emissions in the region of hind paw (bright area) as compared to B. (E) The dynamic changes in biophoton emissions from the hind legs both the treated and control groups at the different time points. Exposure time $=900 \mathrm{~s}$ for each frame image. RGVs: Relative gray values, $\mathrm{n}=7,{ }^{* *}: p<0.01,{ }^{* *}: p<0.001$. 


\section{DISCUSSION}

Rheumatoid arthritis is the most commonly diagnosed systemic inflammatory arthritis, and it carries substantial burden for both the individual and society. For individual, they may suffer from some musculoskeletal deficits, cumulative comorbid risk and quality of life [18]. For society, not only it will cost medical resources, but also, as a consequence of functional disability, it may directly relate to the reduced work capacity and decreased societal participation [19]. However, up to now there are no reliable diagnostic criteria for rheumatoid arthritis [20], and it needs to combine many parameters including the clinical symptoms and the various experimental tests and imaging techniques such as radiological imaging and blood and serology assessment, etc. [21,22], therefore, the early diagnosis and evaluation of the disease process is usually difficult. In this study, we found that the biophoton emission in the hind paws of RA rats was significantly higher than that of the control group, indicating that this novel biophoton imaging technique may give a new way to solve this problem.

Many studies have shown that nonspecific inflammation plays an important role in the development of RA, accompanied by increased formation of free radicals [23]. The generation of free radicals is also an important source of biophotons [10]; therefore, by monitoring the degree of joint biophoton emission, it is not only possible to realize the early diagnosis of RA, but also to monitor the development of disease. In addition, this technique also provides a new method for further using RA animal model to study its pathogenesis and develop new drugs.

\section{ACKNOWLEDGEMENTS}

This work was supported by the research funds of South-Central University for Nationalities (XTZ15014 and CZP 18008).

\section{CONFLICTS OF INTEREST}

The authors declare no conflicts of interest regarding the publication of this paper.

\section{REFERENCES}

1. Cross, M., Smith, E., Hoy, D., Carmona, L., Wolfe, F., Vos, T., Williams, B., Gabriel, S., Lassere, M., Johns, N., Buchbinder, R., Woolf, A. and March, L. (2014) The Global Burden of Rheumatoid Arthritis: Estimates from the Global Burden of Disease 2010 Study. Annals of the Rheumatic Diseases, 73, 1316-1322.

https://doi.org/10.1136/annrheumdis-2013-204627

2. Honda, K. and Littman, D.R. (2012) The Microbiome in Infectious Disease and Inflammation. Annual Review of Immunology, 30, 759-795. https://doi.org/10.1146/annurev-immunol-020711-074937

3. Scher, J.U., Ubeda, C., Artacho, A., Attur, M., Isaac, S., Reddy, S.M., Marmon, S., Neimann, A., Brusca, S., Patel, T., Manasson, J., Pamer, E.G., Littman, D.R. and Abramson, S.B. (2015) Decreased Bacterial Diversity Characterizes the Altered Gut Microbiota in Patients with Psoriatic Arthritis, Resembling Dysbiosis in Inflammatory Bowel Disease. Arthritis \& Rheumatology, 67, 128-139. https://doi.org/10.1002/art.38892

4. Aletaha, D., Alasti, F. and Smolen, J.S. (2015) Rheumatoid Factor, Not Antibodies against Citrullinated Proteins, Is Associated with Baseline Disease Activity in Rheumatoid Arthritis Clinical Trials. Arthritis Research \& Therapy, 17, Article No. 229.

5. Gonzalez, A., Icen, M., Kremers, H.M., Crowson, C.S., Davis, J.M., Therneau, T.M., Roger, V.L. and Gabriel, S.E. (2008) Mortality Trends in Rheumatoid Arthritis: The Role of Rheumatoid Factor. The Journal of Rheumatology, 35, 1009-1014.

6. van Gaalen, F.A., van Aken, J., Huizinga, T.W., Schreuder, G.M., Breedveld, F.C., Zanelli, E., van Venrooij, W.J., Verweij, C.L., Toes, R.E. and de Vries, R.R. (2004) Association between HLA Class II Genes and Autoantibodies to Cyclic Citrullinated Peptides (CCPs) Influences the Severity of Rheumatoid Arthritis. Arthritis and Rheu- 
matism, 50, 2113-2121. https://doi.org/10.1002/art.20316

7. Colli, L., Facchini, U., Guidotti, G., Lonati, R.D. and Sommariva, O. (1955) Further Measurements on the Biolumiescence of the Seedlings. Experientia, 11, 479-481. https://doi.org/10.1007/BF02166829

8. Popp F.A. (2003) Properties of Biophotons and Their Theoretical Implications. Indian Journal of Experimental Biology, 41, 391-402.

9. Popp, F.A., Nagl, W., Li, K.H., Scholz, W., Weingärtner, O. and Wolf, R. (1984) Biophoton Emission. New Evidence for Coherence and DNA as Source. Cell Biophysics, 6, 33-52. https://doi.org/10.1007/BF02788579

10. Cadenas, E. (1984) Biological Chemiluminescence. Photochemistry and Photobiology, 40, 823-830. https://doi.org/10.1111/j.1751-1097.1984.tb04657.x

11. Chilton, C.P. and Rose, G.A. (1984) Urinary Chemiluminescence-An Evaluation of Its Use in Clinical Practice. British Journal of Urology, 56, 650-654. https://doi.org/10.1111/j.1464-410x.1984.tb06138.x

12. Takeda, M., Kobayashi, M., Takayama, M., Suzuki, S., Ishida, T., Ohnuki, K., Moriya, T. and Ohuchi, N. (2004) Biophoton Detection as a Novel Technique for Cancer Imaging. Cancer Science, 95, 656-661. https://doi.org/10.1111/j.1349-7006.2004.tb03325.x

13. Takeda, M., Tanno, Y., Kobayashi, M., Usa, M., Ohuchi, N., Satomi, S. and Inaba, H. (1998) A Novel Method of Assessing Carcinoma Cell Proliferation by Biophoton Emission. Cancer Letters, 127, 155-160.

https://doi.org/10.1016/s0304-3835(98)00064-0

14. Murugan, N.J., Persinger, M.A., Karbowski, L.M. and Dotta, B.T. (2020) Ultraweak Photon Emissions as a NonInvasive, Early-Malignancy Detection Tool: An in Vitro and in Vivo Study. Cancers, 12, 1001. https://doi.org/10.3390/cancers12041001

15. Zhao, X., Yang, M., Wang, Y., Pang, J., van Wijk, E., Liu, Y., Fan, H., Zhang, L. and Han, J. (2017) Spectrum of Spontaneous Photon Emission as a Promising Biophysical Indicator for Breast Cancer Research. Scientific Reports, 7, Article No. 13083. https://doi.org/10.1038/s41598-017-13516-8

16. Usui, S., Tada, M. and Kobayashi, M. (2019) Non-Invasive Visualization of Physiological Changes of Insects during Metamorphosis Based on Biophoton Emission Imaging. Scientific Reports, 9, Article No. 8576. https://doi.org/10.1038/s41598-019-45007-3

17. Tang, R. and Dai, J. (2014) Spatiotemporal Imaging of Glutamate-Induced Biophotonic Activities and Transmission in Neural Circuits. PLoS ONE, 9, e85643. https://doi.org/10.1371/journal.pone.0085643

18. Kitas, G.D. and Gabriel, S.E. (2011) Cardiovascular Disease in Rheumatoid Arthritis: State of the Art and Future Perspectives. Annals of the Rheumatic Diseases, 70, 8-14. https://doi.org/10.1136/ard.2010.142133

19. Sokka, T., Kautiainen, H., Pincus, T., Verstappen, S.M., Aggarwal, A., Alten, R., et al. (2010) Work Disability Remains a Major Problem in Rheumatoid Arthritis in the 2000s: Data from 32 Countries in the QUEST-RA Study. Arthritis Research \& Therapy, 12, Article No. R42. https://doi.org/10.1186/ar2951

20. Smolen, J.S., Aletaha, D. and McInnes, I.B. (2016) Rheumatoid Arthritis. The Lancet, 388, 2023-2038. https://doi.org/10.1016/S0140-6736(16)30173-8

21. Kumar, L.D., Karthik, R., Gayathri, N. and Sivasudha, T. (2016) Advancement in Contemporary Diagnostic and Therapeutic Approaches for Rheumatoid Arthritis. Biomedicine \& Pharmacotherapy, 79, 52-61. https://doi.org/10.1016/j.biopha.2016.02.001

22. Wasserman A.M. (2011) Diagnosis and Management of Rheumatoid Arthritis. American Family Physician, 84, 1245-1252.

23. Hadjigogos, K. (2003) The Role of Free Radicals in the Pathogenesis of Rheumatoid Arthritis. Panminerva Medica, 45, 7-13. 